\title{
Rechtsgeschichte
}

http://www.rg-rechtsgeschichte.de/rg2

Zitiervorschlag: Rechtsgeschichte Rg 2 (2003)

$\operatorname{Rg} 22003 \quad 25-38$

http://dx.doi.org/10.12946/rg02/025-038

\section{Manfred Aschke}

\section{Evolutionstheorie für das Recht der Marktgesellschaft}

Zu Marc Amstutz, Evolutorisches Wirtschaftsrecht. Vorstudien zum Recht und seiner Methode in den Diskurskollisionen der Marktgesellschaft 


\section{Abstract}

As Marc Amstutz shows, contemporary commercial and economic law is characterised by a series of demands which are not easily compatible. To put the problem in theoretical terms: commercial law is required on the one hand to adapt itself to economic needs and circumstances and on the other hand to safeguard its inner logic and autopoiesis. Amstutz tries to escape this dilemma by the figure of "conversation-circles", which allows subsystems to connect within certain boundaries their communications with one another. Thereby the subsystems, such as commercial law, by no means give up their autonomy which is, as Amstutz explains, due to evolutionary mechanisms: the rather firm internal order of systems is exposed to external forces and influences. The system permanently operates at the edge of chaos, a situation by which evolution is made possible. Though Amstutz's work is a great achievement in the theoretical analysis of modern law, what remains unclear is the position of human actors. Amstutz is not successful in building the bridge between social actors and social systems. The crucial problem how "structural coupling" works is not solved. Therefore his arguments concerning the methods of jurisprudence are not entirely convincing. 


\section{Evolutionstheorie für das Recht der Marktgesellschaft}

Zu Marc Amstutz, Evolutorisches Wirtschaftsrecht. Vorstudien zum Recht und seiner Methode in den Diskurskollisionen der Marktgesellschaft*

\section{Die Einheit der Rechtsordnung und die systemtheoretische Eisenbahn}

»Das Rechtsstaatsprinzip verpflichtet alle rechtsetzenden Organe des Bundes und der Länder, die Regelungen jeweils so aufeinander abzustimmen, dass den Normadressaten nicht gegenläufige Regelungen erreichen, die die Rechtsordnung widersprüchlich machen. « ${ }^{\mathbf{I}}$

Das Ideal der Einheit der Rechtsordnung als normatives Argument ist verführerisch und zugleich problematisch. ${ }^{2}$ Je weiter die Vorstellung von der Einheit der Rechtsordnung geht, desto stärker wird der Widerspruch zu unübersehbaren empirischen Befunden einer Fragmentierung der Lebenswelten, einer Pluralisierung und Individualisierung von Werten und einer Heterogenität der Interessen in der Gesellschaft, die der Vorstellung einer Einheit der Rechtsordnung im Sinne einer durchgängigen Homogenität ihrer Dogmatik und ihrer Wertungen den Boden entziehen. Ein besonders skeptischer Blick auf die "Einheit der Rechtsordnung " ergibt sich auf der Grundlage des systemtheoretischen Konzepts der funktionalen Differenzierung der Gesellschaft in Teilsysteme gesellschaftlicher Kommunikation, die jeweils eine eigene Leitdifferenz zu Grunde legen, in diesem Sinne ihre eigene Logik erzeugen und sich selbst steuern, aber nicht von anderen Teilsystemen oder von einem Zentrum oder einer Spitze der Gesellschaft aus steuern lassen.
Auch der Rückgriff auf diese empirischen Befunde und theoretischen Analysen als normatives Argument ist verführerisch und problematisch zugleich. Lässt sich damit eine Überwindung der traditionellen juristischen Methode rechtfertigen, die doch zumindest ganz wesentlich darauf angelegt ist, begriffliche Systematik und innere Stimmigkeit des Rechts zu verbürgen? Juristen sind gewohnt, möglichst auf Widerspruchsfreiheit zu achten. Ein Mindestmaß an Kohärenz des Rechts im Sinne von äußerer und innerer Stimmigkeit ist ein grundsätzlich akzeptiertes Ziel. ${ }^{3}$ Und was sollte an die Stelle dieser auf das Ideal der Einheit der Rechtsordnung verpflichteten traditionellen juristischen Methoden treten? Das Unbehagen gegenüber dem Rückgriff auf systemtheoretische Argumente in der Auslegung von Verfassungsnormen haben kürzlich Pöcker und Wilkens in ihrer kritischen Auseinandersetzung mit der »systemtheoretischen Eisenbahn « sehr deutlich artikuliert. ${ }^{4}$ Der Kommentierung von Vesting zu Art. 87 e GG [Eisenbahnen des Bundes], ${ }^{5}$ die ausdrücklich an systemtheoretische Analysen und an das Konzept der Prozeduralisierung des Rechts anknüpft und daraus Folgerungen für die Auslegung ableiten will, werfen sie u. a. vor, dass die Heranziehung von systemtheoretischen Beschreibungen, die empirisch zudem nicht hinreichend belegt seien - insbesondere die Annahme, bei dem »Eisenbahnwesen « handele es sich um ein Subsystem im Sinne der Systemtheorie,

\footnotetext{
* Baden-Baden: Nomos 200I, 428 S., ISBN 3-7890-76I7-I I BVerfGE 98, I06 (I I 8f.). Zur Debatte um dieses Argument in der Entscheidung des BVerfG zur kommunalen Verpackungssteuer vgl. M. KLOEPFER, K. T. BRÖCKER, Das Gebot widerspruchsfreier Normgebung als Schranke der Ausübung einer Steuergesetzgebungskompetenz nach Art. I05 GG, in: DÖV 200I, I ff.
}

\footnotetext{
2 Vgl. A. Hanebeck, Die Einheit der Rechtsordnung als Anforderung an den Gesetzgeber?, in: Der Staat 4 I (2002) 429 ff., $437 \mathrm{f}$.

3 Vgl. W. Hoffmann-Riem, Kohärenz in der Anwendung europäischer und nationaler Grundrechte, in: EuGRZ 2002, 473.

4 M. Pöcker, S. Wilkens, Die systemtheoretische Eisenbahn, in: Zeitschrift für Gesetzgebung 2002, $334 \mathrm{ff}$.
}

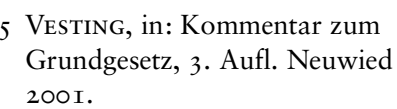

5 Vesting, in: Kommentar zum Grundgesetz, 3. Aufl. Neuwied 2001 . 
das eine eigene systemimmanente Rationalität entfalte -, ähnlich wie der ältere Begründungstopos der »Natur der Sache« letztlich nur zu einem »äußerst flexiblen Umgang mit dem Material « führe, der mit der herkömmlichen Technik und den Standards rechtlichen Argumentierens kaum noch konform sei und weitgehend beliebig wirkende Ergebnisse zeitige. ${ }^{6}$

Normatives Argumentieren mit der Fragmentierung der Lebenswelt und der funktionalen Differenzierung der Gesellschaft wirft also ebenso grundsätzliche Fragen auf wie sein Gegenteil, die normative Argumentation mit der Einheit der Rechtsordnung. Wenn man angesichts der weitreichenden Folgen, die derartige Konzepte haben können, zu verantwortbaren Lösungen gelangen will, braucht man offenbar nicht nur ein hinreichend komplexes Denkmodell für das Verhältnis von Recht und Gesellschaft, sondern auch ein theoretisch reflektiertes Modell für die Entwicklung der »Werkzeuge« rechtlichen Entscheidens. Keine geringere Aufgabe hat Marc Amstutz sich in dem hier zu besprechenden Buch gestellt.

Amstutz geht es um die scheinbar einfache Frage, wie das Recht sich einerseits dem sozialen Wandel aussetzen, zugleich aber seine Eigenlogik bewahren kann. Dafür entwirft er ein theoretisches, operatives und prospektives Modell von hoher Komplexität für das Recht in der Marktgesellschaft. Die Lektüre des Buches erfordert Geduld. Denn Amstutz entwickelt seinen Gedankengang, indem er ältere und neue evolutionstheoretische Konzepte in den Lebenswissenschaften - schon dies ein Verdienst an sich, weil das Buch den tiefen Graben zwischen den Kultur- und den Naturwissenschaften überschreitet, ohne in fragwürdige Reduktionen und Analogien zu verfallen -, in den Sozialwissenschaften und im juristischen Denken auf ihre Verwert- barkeit für das gesuchte komplexe Modell durchmustert. Das Buch entschädigt den Leser reichlich, indem es einen trotz aller Fülle konzentrierten und problemorientierten Überblick über Traditionen und gegenwärtigen Stand evolutionstheoretischen Denkens bietet, der - zumindest in einer für Juristen lesbaren Form nach meiner Kenntnis sonst kaum zu finden ist. Im Folgenden habe ich mich darauf beschränkt, die aus meiner Sicht tragenden Elemente des von Amstutz vorgeschlagenen Modells der Evolution des Rechts in der Marktgesellschaft mit ihrer spezifischen Verbindung von systemtheoretischen und evolutionstheoretischen Argumenten darzustellen und zu diskutieren. Zum Verständnis bedarf es aber zunächst einer etwas genaueren Beschreibung des Themas des wirtschaftsrechtlichen Diskurses, um das es in der weiteren Untersuchung geht.

\section{Der Diskurs des Wirtschaftsrechts}

Als Thema des wirtschaftsrechtlichen Diskurses beschreibt Amstutz die Suche nach dem »ius supra iura ", den Metaregeln, die das gegenseitige Verhältnis jener Rechtsregeln zum Gegenstand haben, die die Marktwirtschaft zu einer sozial durchhaltefähigen Gesellschaftsstrategie machen (I9). Den Grund dafür, dass sich am Anfang des 20. Jahrhunderts ein wirtschaftsrechtlicher Diskurs ausdifferenziert, sieht Amstutz (I6ff.) im Anschluss an die Analyse der »Marktgesellschaft « bei Karl Polanyi in einer »Doppelbewegung«: Das spezifische Moment der neuzeitlichen Marktwirtschaft besteht nicht in der Existenz von Märkten, sondern in dem Anspruch, das Ökonomische in seiner Gesamtheit dem selbstregulierenden Preismechanismus zu unterstellen, was notwendig dazu führe, dass die Wirtschaft nicht mehr eine untergeordnete

6 Pöcker, Wilkens (Rz. 5) $343 \mathrm{ff}$. 
Funktion der Gesellschaft sei, sondern die Gesellschaftssubstanz den Gesetzen des Marktes untergeordnet werde. Das Laissez faire war, darauf weist Amstutz im Anschluss an Polanyi und an die Befunde der Neuen Ökonomischen Soziologie zur Wirtschaftsrechtsgeschichte (I 4 mit Fußnoten 20 und 2I) hin, staatlich geplant und rechtlich institutionalisiert. Aber weil diese Unterordnung der Gesellschaftssubstanz unter die ökonomischen Gesetze des Marktes die Gesellschaft des "Schutzmantels der kulturspezifischen Institutionen « beraubt, der sie vor effets pervers aller Art bewahrt hatte, entsteht eine Gegenbewegung, in der sich soziale Kräfte entfalten, die die Expansion des Marktes zu bremsen versuchen, um die Substanz der Gesellschaft zu schützen. Für das Verständnis dieser sozialen, in Rechtsformen sich niederschlagenden »Gegenbewegung « ist nun entscheidend, dass man sie nicht als Leugnung, sondern als Ermöglichung des Marktes begreift, weil sie die Bedingungen für dessen gesellschaftliche Verträglichkeit schafft. Die Gegenbewegungen sind nicht geplant, sie sind ad-hoc-Reaktionen auf konkrete Effekte des Marktes, die als sozial unverträglich erscheinen. Die Bemühungen um ein Wirtschaftsrecht haben also ihren Grund im unkoordinierten Heranwachsen von verschiedenartigen Normen, denen nur gerade gemeinsam ist, dass sie für den evolutorischen Erfolg der Marktgesellschaft unverzichtbar sind ( 8 f.), weil sie die soziale Durchhaltefähigkeit im Sinne einer Nachhaltigkeit dieser Gesellschaftsstrategie gewährleisten ( $\mathrm{I} 9 \mathrm{f}$.). Als negative Folgeerscheinung kommt es zu Kollisionen und entsprechenden Abgrenzungsfragen. Um welche Art von Kollisionen es dabei insbesondere geht, mag ein Beispiel verdeutlichen, das Amstutz im Kontext seines methodischen Modells beschreibt, das Verhältnis von Kartell- und Markenrecht. Hier

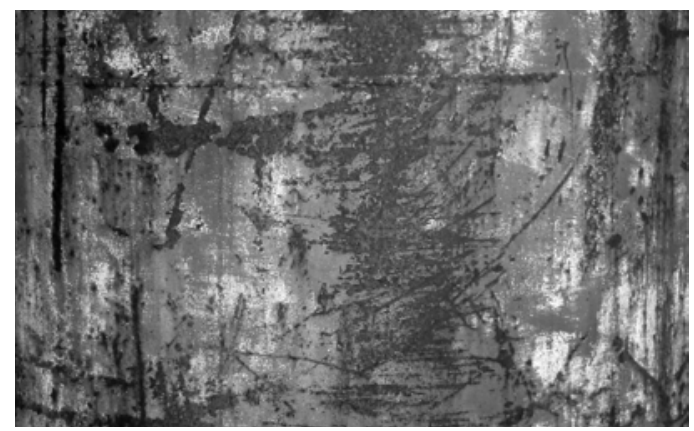

zeigt sich ein diametraler Widerspruch: Die Marke, die das Persönlichkeitsrecht auf freie wirtschaftliche Betätigung konkretisiert, führt zum Ausschluss Dritter vom Gebrauch eines Aussagemittels und schränkt insoweit die rechtliche Betätigungsmöglichkeit der ausgeschlossenen Wettbewerber ein. Da Immaterialgüterrechte, wie Amstutz an anderer Stelle deutlich macht (333), mit dem Wandel zur Wissens- und Informationsgesellschaft neue Aufgaben übernehmen, wird die Problematik deutlich: Lösungen des Konflikts zwischen dem Wettbewerbsrecht, das im Kern ökonomische Funktion hat, und den Immaterialgüterrechten, die im Kern dem Schutz kultureller Werte dienen, die aber auch ökonomisch relevant werden und sich dann im Hinblick auf den Wettbewerb als problematisch erweisen können, sind im Recht nicht schon angelegt und lassen sich auch nicht »ein für allemal« nach einem überzeitlich gültigen Muster gewinnen. Das Recht der Marktgesellschaft ist aber über kurz oder lang darauf angewiesen, intern auf solche Störungen $\mathrm{zu}$ reagieren. Es muss ein gesellschaftlicher "Konsistenztest « durchgeführt werden, der in eine Lehre des juristischen Argumentierens überführt werden kann. Denn nur so wird gewährleistet, dass Konsistenz im Rechtssystem dauerhaft zum Tragen kommt. Das ist für Amstutz das Desiderat, das dem wirtschaftsrechtlichen Diskurs zu Grunde liegt (20).

\section{Zur Charakteristik des theoretischen Grundansatzes der Studie}

Der Grundansatz der Studie, mit der Amstutz in diesen Diskurs eingreift, ist systemtheoretisch und evolutionstheoretisch geprägt. Etwas verkürzt könnte man sagen, dass Amstutz das Problem in der Sprache und im Bezugsrahmen

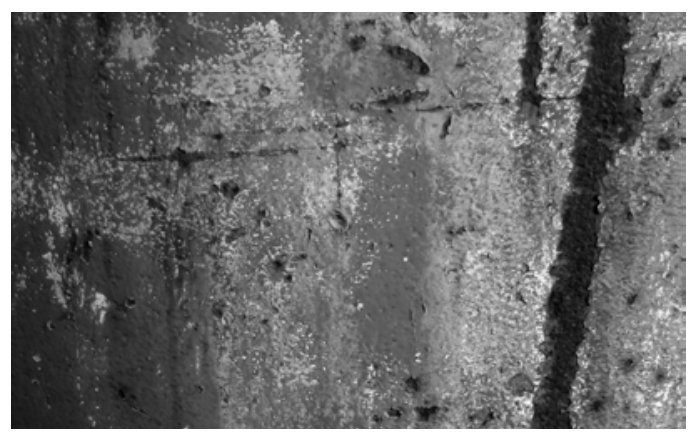


der Systemtheorie formuliert und die möglichen Ansätze für Problemlösungen in der Sprache und im Bezugsrahmen der Evolutionstheorie reflektiert:

Das spannungsreiche Verhältnis zwischen der auf Anpassungs- und Lernfähigkeit eingestellten Dynamik der Marktgesellschaft und dem Recht, das die soziale Durchhaltefähigkeit dieses Gesellschaftsmodells sichert, wird als Problem des Anschlusses der Operationen eines funktionalen Teilsystems, des Rechts, an die Operationen eines anderen funktionalen Teilsystems, der Wirtschaft, beschrieben. Recht muss, um seine konfliktschlichtende Rolle effektiv übernehmen zu können, sich in der einen oder anderen Form an den Binnendiskurs seiner Co-Systeme anschließen. Das Konzept der Autopoiesis, der operationalen Geschlossenheit der funktionalen Teilsysteme, führt hier zu einem Dilemma, das Amstutz als die »bis heute schmerzhafte (weil allen Bemühungen zum Trotz bis heute noch unzulänglich beantwortete) Gretchenfrage « der Theorie sozialer Systeme bezeichnet (55) und mehrfach in unterschiedlichen Formulierungen artikuliert: Wie kann »internalisierte Externalität « organisiert werden? Wie ist die ersichtlich voraussetzungsvolle Verbindung von Selbstbeobachtung und Fremdbeobachtung zu verwirklichen? Auf welche Weise können beide Seiten der Unterscheidung, Recht und gesellschaftliche Umwelt, zueinander ins Verhältnis gebracht werden (54) ? Schließlich: Wie kann das Recht seine Leistungen nach selbst erzeugten Kriterien erbringen und zugleich den vom Markt definierten Bedürfnissen, die in einer Marktgesellschaft die Leitlinien der Gesellschaftsgestaltung definieren, kognitiv-rezeptiv ausgesetzt bleiben (56)?

Anregungen zu einer Lösung dieses Problems gewinnt Amstutz in entscheidendem Maße aus evolutionstheoretischen Konzepten, die in den Lebenswissenschaften entwickelt und diskutiert worden sind (Konzept der ökologischen Nische, Theorie der unterbrochenen Gleichgewichte, das NK-Modell S. A. Kauffmans), die er aber nicht im Wege der Transformation oder der Analogie verwertet, sondern als »Metaphern « nutzen will, als Bilder, die die Suche nach ähnlichen Lösungswegen in den Sozialwissenschaften anregen.

\section{Die Erkenntnistheorie des »sozial-kognitiven Konfigurationismus «}

Einen grundlegenden Ausgangspunkt für das Modell des Rechts in der Marktgesellschaft bildet für Amstutz die Erkenntnistheorie des "sozial-kognitiven Konfigurationismus « (6I ff.). Damit ist eine erkenntnistheoretische Grundannahme gemeint, die Amstutz nahezu deckungsgleich bei von Hayek in dessen Lehre von der »abstrakten Ordnung " und in den Aussagen der Rotterdamer Schule des Konfigurationismus findet: In sozialen Austauschprozessen oder »double interacts « kommt es zu einer gegenseitigen Abstimmung der erkennenden Subjekte bzw. zu einer von beiden Akteuren geteilten Realitätsumschreibung. Die erkennenden Akteure bewegen sich in der Regel gleichzeitig in mehreren »Konfigurationen«. Sie bringen Wirklichkeitskonstruktionen, die einer bestimmten Konfiguration entstammen, regelmäßig in andere Konfigurationen ein. Vor diesem Hintergrund deutet Amstutz die Lehre von der "abstrakten Ordnung «: Für von Hayek steht außer Zweifel, dass Regeln und damit auch Recht für sich allein noch lange keine Seinsordnung begründen. Eine solche wird vollständig immer erst durch die besonderen Absichten und Tatsachenkenntnisse der Handelnden bestimmt. Die Schaffung einer Seinsordnung hängt deshalb für von Hayek nicht 
nur von Rechtsregeln oder anderen normativen Faktoren ab, sondern auch von tatsächlichen Umständen, auf die die Rechtsregeln hinweisen. Von Hayek unternimmt auf dieser Grundlage eine evolutionstheoretische Deutung der Auflösung von Rechtskollisionen: Fragen der Abstimmung von Normen, die in der "Konfiguration Recht " auftauchen, werden in die "Konfiguration Handeln « überführt. Dort ergibt sich die Antwort dank "natürlicher Selektion « in der Weise, dass die Handlungsträger, wenn auch meistens unbewusst, aus allen möglichen Varianten von Normabstimmungen jene auswählen, die der Gruppe, der sie angehören, am meisten nützen. Amstutz hebt positiv hervor, dass von Hayek damit der Anschluss des Rechts an andere "social fields « gelinge, er kritisiert aber, dass die bei von Hayek unterstellten Bezüge zwischen Rechtsordnung und Handelnsordnung nicht differenziert genug modelliert werden, mit der Konsequenz, dass seine Lehre in einen einseitigen juristischen Adaptionismus münde, der die Rationalität des Rechtssystems bedroht. Den Anspruch des gesuchten komplexen Modells beschreibt Amstutz dagegen folgendermaßen: "Die Operationen des Rechtssystems sind (...) keineswegs gleichartig, sondern unterscheiden sich ihrer Natur nach mitunter erheblich; so sind Sachverhalte zu erstellen, Rechtspolitiken zu definieren, dogmatische Figuren zu konstruieren usw. Ein >Modell-von-Recht-in-Gesellschaft muss imstande sein, anzugeben, wie Wirklichkeit $<$ in diese heterogenen Operationen einfließen soll. Davon hängt letztlich die Fähigkeit des Rechts $\mathrm{ab}$, seine Identität $\mathrm{zu}>$ organisieren $<(.$.$) «$ (65).

Aus einem theoretischen Modell der Marktgesellschaft gewinnt Amstutz die Schlussfolgerung, dass der Aufbau eines "Schutzmantels kulturspezifischer Institutionen «, der die Markt-

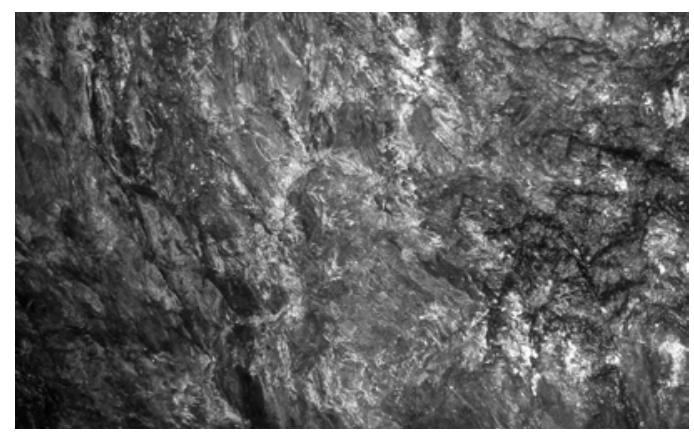

wirtschaft als gesellschaftliche Strategie durchhaltbar macht, ein laufender, dauerhafter, nie endender Prozess ist. Auf zeitunabhängige »Ein-für-allemal«-Lösungen bei der Abstimmung von marktgesellschaftlichen Normen muss verzichtet werden. Dazu muss auf der MetaEbene eine »bewegliche Verfassung « gesucht werden, die die Mechanismen der Konfliktlösung an den temporalisierten Kreislauf der kollidierenden Normen sozusagen anschließt und mit der nämlichen Dynamik versieht, indem sie die Aufgabe der Kollisionslösung ebenfalls evolutionären Mechanismen anvertraut $(82,83)$.

Eine Leitfrage der weiteren Untersuchung lautet: Wie erlangt das Rechtssystem Kenntnis von den Ordnungsbedürfnissen anderer sozialer Teilsysteme und wie wird diese Information vom Rechtssystem verwertet, d. h. in rechtliche (Ordnungs-)Leistungen umgesetzt? (2 I7) Einen wichtigen Impuls für die Untersuchung dieser Frage gewinnt Amstutz aus Teubners These der (zunehmenden) Unbestimmtheit des Rechts. Sie weist auf zwei Gesichtspunkte hin (2I8). Erstens: Recht tritt stets als »Ordnungsentwurf « in Kraft, als Prototyp eines sozialen Designs, der, um seine Funktion erfüllen zu können, noch der Adaptation an seine gesellschaftliche Umwelt bedarf. Zweitens: Diese Adaptation kann weder ausschließlich rechtsintern noch schlagartig erfolgen. Sie nimmt sich viel eher als eine Art »tâtonnement «-Prozess aus, in dem das Rechtssystem in wiederkehrenden Diskursschlaufen mit anderen Sozialsystemen seine Ordnungsleistungen allmählich an die Bedürfnisse der Gesellschaft anzupassen sucht. Recht "geschieht « in erster Linie im Rahmen dieses systemübergreifenden Vorgangs, es hat seinen Schwerpunkt also, entgegen Eugen Ehrlich, nicht in der Gesellschaft allein, sondern in dieser und im Rechtssystem zugleich.

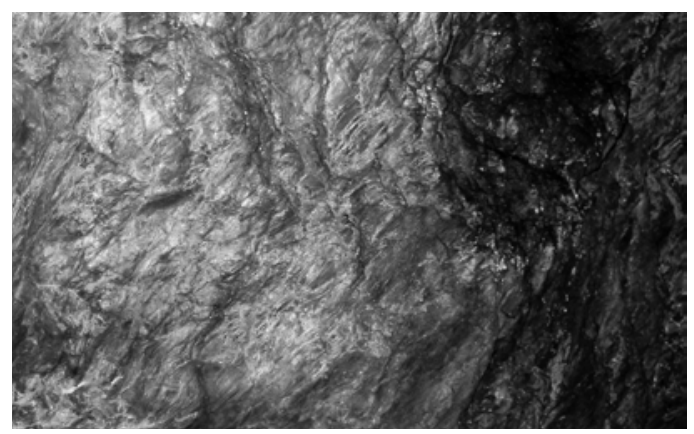


Von diesem Ansatz aus kann Amstutz zunächst die Gedanken von Hayeks aufgreifen, der Rechtsordnung (als abstrakte Ordnung) und Handelnsordnung (als konkrete Ordnung) unterscheidet und Recht als dreipoligen Interaktionsprozess zwischen Rechtsregeln, Akteuren (Handlungen) und Umständen (konkrete Tatsachen des Augenblicks) versteht. Amstutz sieht darin einen intuitiv richtigen, aber theoretisch noch unterentwickelten Ansatz: Unklar bleibe die eigentliche Natur der drei Elemente, wie sich die Interaktionen zwischen ihnen im Einzelnen gestalten, wie Rechtsnormen, Handelnde und Umstände miteinander verknüpft sind, was genau die doch etwas mysteriöse Handelnsordnung ist und wo ihr konkreter »Platz « in der Gesellschaft ist. Für die theoretische Weiterentwicklung des von Hayekschen Ansatzes zieht Amstutz nun die von Hutter entwickelte Theorie der Konversationskreise heran, die er allerdings generalisiert und radikalisiert (22 I). Damit kommen wir zu der ersten zentralen Säule des Modells, das Amstutz vorschlägt.

\section{Konversationskreise als autopoietische} Systeme

Hutter $^{7}$ stellte sich die Frage, wie es der Wirtschaft gelingt, die rechtlichen Bedingungen des Wirtschaftens anzupassen, wenn innerhalb der Wirtschaft Veränderungen (zum Beispiel technische Innovationen mit einem neuen Horizont der Wertschöpfung) entstanden sind. Seine zentrale These lautet: Wenn gesellschaftliche Teilsysteme als »Mitteilungsströme « zu interpretieren sind, liegt es nahe, dass auch in der Kommunikation zwischen Wirtschafts- und Rechtssystem soziale Formen entstehen, durch die Mitteilungen für beide Systemtypen besser formuliert und besser verständlich werden. Mit
Hilfe von »Konversationskreisen « können die Subsysteme der Gesellschaft die Probleme überwinden, die ihre autopoietische Geschlossenheit verursacht. Sie ermöglichen intersystemische Kommunikation. Für Amstutz sind solche Konversationskreise evolutionäre Errungenschaften, die in einer funktional ausdifferenzierten Gesellschaft den "kommunikativen Anschluss « des Rechtsdiskurses an den Diskurs anderer Sozialsysteme fördern. Zwischen dem Recht und anderen gesellschaftlichen Teilbereichen nistet sich eine Myriade von Konversationskreisen ein, die alle eigenständige »kleine " soziale Systeme bilden. In der Verdeutlichung seiner These macht Amstutz darauf aufmerksam, dass die eigentliche List der Idee der Konversationskreise darin liegt, direkte und damit unwahrscheinliche (weil informationell überladene) Interaktionen zwischen den großen funktionalen Systemen der Gesellschaft, wie sie in der Theorie des Ultrazyklus von Teubner vermutet werden, zu vermeiden und statt dessen bescheidenere, aber aussichtsreichere (weil einfacher zu handhabende) Beziehungen zwischen einzelnen Teildiskursen, die sich innerhalb dieser großen Funktionssysteme abspielen, herzustellen. Konversationskreise sind weder Subsysteme des Rechts noch irgendeines anderen großen funktionalen Sozialsystems, sondern sie entstehen unmittelbar in der Gesellschaft, auf die sich auch ihre spezifische Leistung bezieht. Der »Clou « der Lösung aber, die Amstutz vorschlägt, besteht darin, dass die Konversationskreise selbst als kleine, "kapillare " autopoietische Systeme angesehen werden, die nicht durch »multiple Leitdifferenzen « (Hutter), sondern durch die eigene Leitdifferenz »Dialog / Monolog « codiert werden. Konversationskreise sind dann wechselseitig an Recht und regulierten Sozialbereich strukturell gekoppelt. Es entsteht ein Kreislauf Recht / Konversationskreis / reguliertes Sozial-

\footnotetext{
7 Michael Hutter, Die Produktion von Recht. Eine selbstreferentielle Theorie der Wirtschaft, angewandt auf den Fall des Arzneimittelpatentrechts, Tübingen I989.
} 
system, der in wiederholten Schlaufen die Abstimmung zwischen Recht und reguliertem Sozialbereich bewerkstelligt (siehe dazu das Schema auf S. 24I).

Evolutionstheoretische Plausibilität für dieses Konzept der Konversationskreise gewinnt Amstutz (246 ff.) aus dem Konzept der ökologischen Nische und insbesondere aus der Unterscheidung von fundamentaler und realisierter Nische (Hutchinson). Theoretisch überschneiden sich die ökologischen Nischen unterschiedlicher Tier- und Pflanzenarten, d. h. die Ausschnitte der Umwelt, in denen sie die erforderlichen Ressourcen zum Überleben finden. Im Prozess der Realisierung der ökologischen Nische, die von einer bestimmten Art besetzt wird, kommt es zur wechselseitigen Abstimmung zwischen der Population einer Tier- oder Pflanzenart und »ihrer« ökologischen Nische. Dabei prägt die Art auch ihrerseits ihre Umwelt, indem sie zum Beispiel das Nährstoffangebot für andere Tieroder Pflanzenarten vermehrt oder verringert. Im Prozess der Realisierung der ökologischen Nische findet zugleich ein Wettbewerb um die Nutzung der vorhandenen Ressourcen und damit auch eine natürliche Auslese statt. Das Resultat kann ein langfristig stabiles homöostatisches Gleichgewicht in dieser Beziehung zwischen der Art und ihrer realisierten ökologischen Nische sein. Eine ähnliche Abstimmung erfolgt nach der Idee von Amstutz auch zwischen den Ordnungsentwürfen des Rechts und den Ordnungsbedürfnissen der regulierten Sozialsysteme. Die Abstimmung erfolgt gewissermaßen in einem Prozess der »Realisierung der ökologischen Nische" in der Umwelt des Rechts, also nicht " auf höchster Ebene «, sondern anhand der Lösung konkreter Probleme. Dabei hilft die »Myriade « von »kapillaren « Konversationskreisen. Allerdings bleibt zu klären, auf welche Ein- heiten sich die dabei stattfindende Selektion bezieht.

\section{Interne Faktoren der Evolution und Eigenlogik des Rechts}

Ging es bisher um externe Faktoren der Evolution des Rechts in der Marktgesellschaft, so wendet sich Amstutz nun den internen Faktoren zu. Es geht um die Frage, wie das Recht seine Eigenlogik durchhält. »Anpassung « allein brächte die Gefahr mit sich, dass das Recht seine Eigenlogik preisgibt. Die Leistungen des Rechts werden aber in anderen Funktionssystemen der Gesellschaft nur nachgefragt, wenn das Recht seine Eigenlogik wahrt und damit Ressourcen für die Lösung von Konflikten anbieten kann, über die andere Funktionssysteme nicht verfügen.

Amstutz greift hier die Theorie der unterbrochenen Gleichgewichte (punctuated equilibria) auf, die im gegenwärtigen Diskurs um eine Modifikation oder zumindest Ergänzung der Synthetischen Theorie besonders einflussreich ist und allgemeine Gesichtspunkte zutage gefördert hat, die auch für die Geisteswissenschaften bedeutsam sind (258ff.). Diese Theorie geht davon aus, dass die Befunde der Fossiliengeschichte, die äußerst selten ganze Serien von Übergangsformen in den tierischen und pflanzlichen Fossilien aufweist, nicht auf Unvollständigkeit und Lückenhaftigkeit beruhen, sondern dass sie ein korrektes Abbild des Evolutionsgeschehens liefern. Danach ergibt sich ein Bild der Evolution, nach dem große evolutionäre Ereignisse wie die erfolgreiche Bildung einer neuen Art während kurzer Schübe der Evolution stattfinden. Vorherrschend sind aber lange Perioden des Stillstandes (Stasis, homöostatisches Gleichgewicht), die manchmal Jahrmillionen dauern 
können und in deren Verlauf es nur zu minimalen Veränderungen kommt.

Der Punktualismus wirft nun aber die Frage auf, wie das Phänomen der Stasis über lange Zeiträume, die eiszeitliche Zyklen und damit drastische Umweltveränderungen einschließen, angesichts der kontinuierlichen Wirkung der natürlichen Auslese erklärt werden kann. Weder die Idee einer stabilisierenden Wirkung von Selektion noch optimale Anpassung liefern überzeugende Gründe. Durchgesetzt hat sich die Ansicht, dass epistatische Interaktionen zwischen Genen (genetische Homöostase) die Ursache von Stasis ist. Über dieses Phänomen weiß man noch verhältnismäßig wenig. Gesucht wird nach den »constraints ", die der Formbarkeit von Organismen durch natürliche Auslese eine Grenze setzen. Da der Punktualismus zwar am Gradualismus, nicht aber am Prinzip der natürlichen Auslese Anstoß nimmt (269), wird eine grundlegende Sichtweise vorgeschlagen, nach der darwinistische Selektion die Theorie ist, die die "normale« Ursache von Evolution beschreibt. Auf historischen oder formalen (strukturellen) Gründen beruhende constraints dämmen im Prozess der Evolution die Wirkung der natürlichen Auslese ein. Zusätzlich zu den externen Faktoren der Evolution, die Gegenstand der Theorie der darwinistischen Selektion sind, müssen also interne Faktoren der Evolution als evolutionsgestaltende Kräfte in Rechnung gestellt werden.

Das NK-Modell Stuart A. Kauffmans und die Teilrechtsordnung als "unit of selection "

Wesentliche Einsichten über das Verhältnis zwischen Selbstorganisation und Selektion gewinnt Amstutz (278 ff.) bei Stuart A. Kauffman, der die Stabilität genetischer Systeme mit Hilfe von iterativen Computerprogrammen untersucht hat, die mit Booleschen Netzwerken als Modellen des genetischen Systems arbeiteten. Nach dem NK-Modell Kauffmans entscheidet zuerst die innere Ordnung der unter Selektionsdruck stehenden Einheit über die Evolutionsfähigkeit des Systems. Die Selektion gibt ihre Rolle als Kreator auf und wird zu einer Art Suchmaschine: Sie bringt innerlich bereits adäquat organisierte Einheiten dorthin und versucht sie dort zu halten, wo ihre Evolutionsaussichten optimal sind: am Rande des Chaos. Selektion kann, so lautet die Schlussfolgerung, nicht aus eigener Kraft Evolution in Gang bringen. Erst das Zusammenwirken von Selektion mit Selbstorganisation macht Evolution möglich.

Das im NK-Modell Kauffmans aufgedeckte Interaktionsverhältnis von internen und externen Evolutionsfaktoren will Amstutz nun als Metapher für die Suche nach dem operativen Modell der Evolution des Rechts in der Marktgesellschaft nutzen. Seine These lautet (288):

Die Evolutionsfähigkeit des Rechtssystems wird gewährleistet durch »Teilrechtsordnungen ", die ihre »Nischen" in der gesellschaftlichen Umwelt des Rechts definieren. Zwischen den einzelnen Elementen dieser » Teilrechtsordnungen « (Prinzipien, positive Normen, Richtersätze, Gewohnheitsrecht usw.) kristallisiert sich als spontane Ordnung ein »Beziehungszusammenhang « heraus, ein regulatorisches Netzwerk, das eine »kohärente und ästhetische Geschichte « bildet und dadurch Evolutionsfähigkeit schafft. Amstutz nennt diesen Beziehungszusammenhang »teilrechtsordnungsspezifische Policy«. Sie ermöglicht erst, dass die Teilrechtsordnung externen selektiven Kräften ausgesetzt wird und in den Perturbationen der iterativen Schlaufe Recht / Konversationskreis / reguliertes Sozialsystem »Fitness « (Gesellschaftsadäquität) er- 
wirbt, ohne den Preis des Verlustes ihrer Eigenlogik zahlen zu müssen.

Amstutz sucht nach dem »epistatischen Sinnzusammenhang “, der spontan, also nicht erst unter dem selektiven Einfluss der Perturbationskreisläufe »Recht / Konversationskreise / regulierte Sozialsysteme « entsteht und in dem sich die Elemente der Teilrechtsordnung in einem ähnlichen Sinne verknüpfen, wie das im interaktiven Prozess zweier oder mehrerer Gene der Fall ist, der für die Züge eines bestimmten Individuums ursächlich ist (294). Das »interne Ordnungsprinzip « der Teilrechtsordnung, die »Lokalisierung ihrer Regelungsaufgabe in der Gesellschaft«, ergibt sich aus der »künftigen Situation in operationaler Beschreibung «. Es geht um die "Suggestion eines Netzes von abgleichenden (regulierenden) Interaktionen zwischen den einzelnen Elementen einer gegebenen Teilrechtsordnung ", um den bei der Genese der Teilrechtsordnung, in der Herausbildung einer Praxis spontan entstehenden »kohärenzstiftenden Beziehungszusammenhang « zwischen Elementen der Teilrechtsordnung.

Diese »Policies « von Teilrechtsordnungen sind unverfügbar, "evolutionsfest « im Sinne der Stasis des Punktualismus. Ihre Elemente aber sind »plastisch «. Das ermöglicht Adaptation an die Umwelt - bis zu der durch die Policy beschriebenen Grenze. Die Elemente fungieren gewissermaßen als Rezeptoren für die Perturbationen der Außenwelt und schaffen so die Möglichkeit, dass Selektion Teilrechtsordnungen dorthin treibt, wo sie am funktionsfähigsten. In diesem Prozess lösen sich auch die Kollisionen der Teilrechtsordnungen auf, »indem ihre jeweiligen Elemente, dort wo sie konfligierend aufeinandertreffen, evolutorisch kompatibilisiert werden $«(296)$.

\section{Einwände}

In der gründlichen Auswertung neuester Diskussionen und Ergebnisse der Evolutionsforschung in den Lebenswissenschaften auf verwertbare Metaphern für die Sozialwissenschaften liegt ohne Zweifel der kaum zu überschätzende Wert der Studie von Amstutz. Das gilt insbesondere für die plausibel begründete Hypothese, dass auch soziale Systeme nur evolutionsfähig sind, wenn interne und externe Faktoren zusammenspielen. Das gilt auch für die Unterscheidung von fundamentaler und realisierter ökologischer Nische und für die These, dass die Art von interner Ordnung, die Evolutionsfähigkeit ermöglicht, durch »low connectivity«, durch lose Verknüpfung ihrer Elemente bedingt ist, die dem System einen Zustand von »Ordnung am Rande des Chaos" verleihen. Meine Einwände richten sich gegen die Umsetzung im Modell des Rechts in der Marktgesellschaft.

In der Argumentation von Amstutz fällt ein Widerspruch auf. Einen erkenntnistheoretischen Ausgangspunkt der Studie, der auch später wieder aufgegriffen wird ( 2 I 9 ff.), bildet der »sozialkognitive Konfigurationismus « mit von Hayeks Vorstellung eines dreipoligen Interaktionsprozesses zwischen Rechtsregeln, Akteuren (Handlungen) und Umständen (konkreten Tatsachen des Augenblicks). Amstutz kritisiert, dass die eigentliche Natur dieser Elemente und ihre Verknüpfung unklar seien. Er will den »intuitiv richtigen, aber theoretisch unterentwickelten Ansatz « von Hayeks theoretisch rekonstruieren (220 f.). Dann muss aber die Frage gestellt werden, wo im letztlich vorgeschlagenen Modell die "Akteure « bzw. deren »Handlungen « bleiben oder was mit gleicher oder besserer theoretischer Leistung an deren Stelle tritt. Amstutz setzt sich 
zwar kritisch mit dem Versuch einer akteurtheoretischen Ergänzung des autopoietischen Ansatzes in der Theorie der Verhandlungssysteme des Kölner Max-Planck-Instituts für Gesellschaftsforschung auseinander (23 I ff.). Aber er zeigt weder eine Alternative noch ein theoretisches Äquivalent auf. Nehmen die Teilrechtsordnungen oder ihre "Policies « die Stelle der Akteure ein? Was wird dann aber aus dem für die Konzeption von Hayeks doch so entscheidenden verstreuten Wissen, den besonderen Absichten und Tatsachenkenntnissen der Handelnden? Und was wird aus der Einbeziehung der Akteure in unterschiedliche soziale Konfigurationen? Sind die Akteure Teil der »ökologischen Nische", die das Recht in Gestalt von Teilrechtsordnungen besetzt? Wie lassen sich dann die Beziehungen zwischen Teilrechtsordnungen und Akteuren als deren Umwelt theoretisch modellieren? Auf diese Fragen findet man bei Amstutz weder ausdrücklich noch in der Sache eine befriedigende Antwort. Das handelnde Individuum taucht erst im abschließenden Methodenkapitel wieder auf, als vorausgesetztes Erkenntnis- und Entscheidungssubjekt.

Mit der vergleichsweise jungen Tradition der Systemtheorie ist die Vorstellung einer »Interaktion « zwischen Normen, Akteuren und äußeren Umständen unvereinbar. Soziales System und Bewusstsein werden gerade als operational geschlossene Systeme konzipiert. »Interaktion « im Sinne einer Verknüpfung von Operationen gibt es nicht. An deren Stelle treten das Konzept der "strukturellen Kopplung " und die eher vage Vorstellung der Entstehung von Ordnung aus Lärm (»order from noise«). Amstutz sieht deutlich, dass die mit diesen Konzepten verbundenen Fragen noch nicht befriedigend gelöst sind. Um so mehr durfte man gespannt sein, wie er die Anregungen des sozial-kognitiven Konfigura- tionismus system- und evolutionstheoretisch aufnehmen und integrieren kann. Im Zentrum seines Entwurfs stehen die autopoietischen Konversationskreise. Aber mit ihnen wird die »Gretchenfrage « der Systemtheorie nicht gelöst, sondern (nur) von der Ebene der großen Funktionssysteme der Gesellschaft auf die Ebene der Myriade kapillarer Übersetzungsinstitutionen verschoben. Wenn diese Konversationskreise im gleichen Sinne autopoietische Systeme sind wie die Funktionssysteme Wirtschaft, Politik, Recht usw., bleibt rätselhaft, wie sie das Ergebnis ihrer Operationen nach der Leitunterscheidung "Dia$\log /$ Monolog « den jeweiligen Funktionssystemen, die ein Abstimmungsproblem haben, mitteilen können. Denn jene verstehen doch nur ihre eigene Sprache und die Operationen der Konversationskreise bleiben für sie genauso Lärm wie die Operationen anderer Teilsysteme - es sei denn, es bestünden bereits »strukturelle Kopplungen«. Aber darum geht es ja gerade, wie solche strukturellen Kopplungen aus Anlass neu auftauchender Abstimmungsprobleme entstehen.

Gleichwohl halte ich die Verortung der Suche nach einer Lösung auf der Ebene anlassbezogener konkreter Abstimmungsprobleme und die Metapher der fundamentalen und der realisierten Nische bei Amstutz für einen beachtlichen theoretischen Gewinn. Denn diese Schritte ermöglichen eine Präzisierung der »Gretchenfrage « der Systemtheorie mit evolutionstheoretischen Argumenten. Die evolutionstheoretische Metapher der fundamentalen und der realisierten Nische deckt sich in einem wesentlichen Aspekt mit der Unterscheidung zwischen abstrakter und konkreter Ordnung bei von Hayek: Sowohl das genetische Programm als auch der Ordnungsentwurf des Kommunikationssystems Recht bedürfen einer Umsetzung in eine neue 
Dimension, in der die Auseinandersetzung mit den konkreten Gegebenheiten der Umwelt und der Wettbewerb mit konkurrierenden Programmen erfolgen kann. Auf der Sinnebene der Kommunikationssysteme selbst sind solche Prozesse ebenso wenig vorstellbar wie auf der Ebene der genetischen Information. Das genetische Programm bedarf der Umsetzung durch die Proteinbiosynthese zum Aufbau eines Organismus, der dreidimensionale räumliche Strukturen aufweist und dem physischen Einfluss der Umwelt ausgesetzt ist. ${ }^{8}$ Erst auf dem Weg über die konkreten individuellen Organismen kann das genetische Programm einer Tier- oder Pflanzenart seine ökologische Nische realisieren und andere Tierund Pflanzenarten aus seiner ökologischen Nische verdrängen oder in sie integrieren. Offensichtlich kann auch der epigenetische Zusammenhang einzelner Gene in seinem Informationsgehalt nur verstanden werden, wenn man seine Funktionen für den Aufbau des Organismus in die Betrachtung einbezieht.

In der Grundkonzeption der Synthetischen Theorie der biologischen Evolution ${ }^{9}$ nimmt denn auch der Organismus die unverzichtbare Funktion der Einheit der Selektion wahr. Die Organismen realisieren das genetische Programm und die ökologische Nische der Art. Sie sind deshalb der Ansatzpunkt für die selektive Wirkung der Umwelt. Die Selektion wirkt nicht im Sinne einer direkten Veränderung des individuellen Genoms, sondern durch Modulation der Wahrscheinlichkeit, mit der die individuellen Genotypen im Genpool der Art repräsentiert sind. Einheit der Evolution ist deshalb auch weder das Gen oder das Genom noch der Organismus, sondern der Genpool einer Population. ${ }^{\text {Io }}$

Sucht man bei Amstutz nach einer entsprechenden Konzeption für die Evolution sozialer Systeme, stößt man auf Unklarheiten: Teil- rechtsordnungen sind die "units of selection ", »die evolvieren und dem Selektionsprozess der Rechtsevolution unterliegen « (290). Ihnen wird der »Selbstorganisationswert « zugesprochen, der auf den epistatischen Verknüpfungen von Elementen zu einer »Policy « beruht und der sie zu evolutionsfähigen Einheiten macht. Aber Teilrechtsordnungen sind lediglich Ausdifferenzierungen des Kommunikationssystems Recht. Ihnen fehlt die Umsetzung in die Dimensionen, in denen Umwelteinflüsse selektiv wirken können. Auch die angenommene epistatische Verknüpfung von Elementen der Teilrechtsordnung zur »Policy « kann ohne Bezugnahme auf ihre Funktionen für einen sozialen Zusammenhang, der auf einer anderen Ebene als der des Kommunikationssystems Recht liegt, nicht verstanden werden. Die Einheit der Selektion kann auch nicht zugleich die Einheit sein, die evolviert. Das spezifische Moment der evolutionstheoretischen Konzeption der Wirkung der Umwelt auf das genetische System, das auf Darwins Gedanken der natürlichen Auslese zurückgeht und das auch durch den Punktualismus nicht in Zweifel gezogen wird, besteht gerade darin, dass an Stelle einer deterministischen Beziehung eine nur mittelbar wirkende Einflussnahme durch differenzielle Selektion angenommen wird, die die Variationsbreite der im Genpool einer Art repräsentierten individuellen Genotypen moduliert. Für die Systemtheorie, die die Annahme deterministischer Beziehungen zwischen System und Umwelt verwirft, sollte dieses evolutionstheoretische Konzept der externen Selektion attraktiv sein. Dazu bedürfte es allerdings einer Überwindung der bisher gültigen Vorstellung, Systemtheorie sei nur mit interner Selektion vereinbar. Statt die Evolutionstheorie den axiomatischen Vorgaben des Autopoiesebegriffs anzupassen, könnte es hilfreich sein, die Systemtheorie in
8 Vgl. dazu B.-O. Küppers, Der Ursprung biologischer Information. Zur Naturphilosophie der Lebensentstehung, München u. a. I986, $50 \mathrm{ff}$.

9 Siehe dazu M. Aschke, Kommunikation, Koordination und soziales System. Theoretische Grundlagen für die Erklärung der Evolution von Kultur und Gesellschaft, Stuttgart 2002, $26 \mathrm{ff}$. m. w. N.

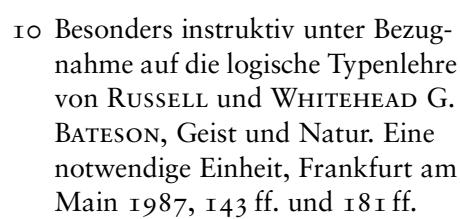

Besonders instruktiv unter Bezugnahme auf die logische Typenlehre von Russell und Whitehead G. Main $1987, \mathrm{I} 43$ ff. und I $8 \mathrm{Iff}$ 
ein schlüssiges evolutionstheoretisches Konzept einzupassen, das externe Selektion einschließt. ${ }^{\text {II }}$ Dafür spricht sich Amstutz auch aus (siehe etwa II3 f., 278, 282). Sein Modell geht diesen Weg aber nicht konsequent genug. Dazu mag die Vorentscheidung beigetragen haben, für sein Modell alle als darwinistisch qualifizierten Evolutionstheorien einschließlich der Synthetischen Theorie, die zu Unrecht mit der Soziobiologie identifiziert wird ( $185 \mathrm{ff}$.), a uszuscheiden ( $2 \mathrm{I} \circ \mathrm{f}$.), obwohl auch die Theorie der unterbrochenen Gleichgewichte den Gedanken der natürlichen Auslese und das Grundkonzept der Synthetischen Theorie nur modifiziert und nicht im Ganzen verwirft.

\section{Juristische Methode und Erfahrung}

Juristische Methode ist Anleitung für individuelle Akteure, insbesondere für Richter, bei der Erkenntnis des Rechts und bei der Gewinnung rechtlicher Entscheidungen. Die Theorie autopoietischer Systeme muss sich mit einem solchen Anspruch schwer tun, solange sie für die Verbindungen zwischen sozialem System und individuellen Akteuren nicht mehr anbieten kann als die Vorstellung, dass aus Lärm Musik werden kann. Es ist eben bisher nicht klar, welche Rolle der Richter für die Autopoiesis des Rechts spielt. Auch im Modell von Amstutz fehlt eine Einheit der Selektion, die das Kommunikationssystem Recht in eine Dimension übersetzen könnte, in der die Umwelt des Rechts einschließlich der handelnden sozialen Akteure im Sinne differenzieller Selektion wirksam werden kann. Dieser Fehlstelle im operativen Modell entspricht spiegelbildlich eine Fehlstelle im Methodenkapitel. Hier erscheint der Richter unausgesprochen wie weitgehend in der traditionellen Methodenlehre - als souveränes Subjekt der Erkenntnis, ohne theoretische Reflexion der kommunikativen und sozialen »constraints «, die individuelle Erfahrung und Erkenntnis erst ermöglichen, aber zugleich auch begrenzen.

Am Beginn des Methodenkapitels wird ein idealtypischer Gegensatz zweier erkenntnistheoretischer Grundpositionen behauptet, des Rationalismus, dem die traditionelle Methodenlehre zugeordnet wird, und des Empirismus, dem das von Amstutz gesuchte "prospektive Modell « zugeordnet wird (307). Der Weg, Rechtsfindung durch Rückgriff auf Erfahrungen zu betreiben, birgt allerdings eine Gefahr, die Amstutz mit einem Zitat von Josef Esser klar benennt: die Gefahr einer dogmatisch unkontrollierten Entwicklung einer subjektiv wertenden, naturrechtlich oder sozialethisch eingestellten Judikatur, die das Systemgebäude der konstruktiven Jurisprudenz eines Tages unbewohnt und verödet liegen lässt. Amstutz hält es gerade für die Funktion einer Methodenlehre, »das Recht davor zu bewahren, dass es unter dem (sehr realen) Druck hegemonischer Sozialinteressen klein beigibt « (3 I9). Amstutz greift die im operativen Modell herausgearbeitete Bedeutung der Eigenlogik des Rechts auch unter methodischen Aspekten auf und stellt klar, dass es nicht darum gehen kann, die traditionelle juristische Methode insgesamt durch eine ausschließlich erfahrungsgestützte Methode und normatives Argumentieren pauschal durch den Verweis auf die Eigendynamik evolutorischer Prozesse zu ersetzen (3 I9 ff.). Der Einbau von Erfahrung, der die Anknüpfung an die evolutorisch-fluktuierenden Bedürfnisse der regulierten Sozialsysteme zur Bewältigung von Kollisionsfragen ermöglichen soll, betrifft, wie Amstutz im Anschluss an die kritische Analyse der Fallnormentheorie Fikentschers (3 I9 ff.) und an die kollisionsrechtliche Methode des IPR (329 ff.) feststellt, nur einen Teilaspekt der Kol-

I I Vgl. Aschke (Fn. 9), insbesondere $284 \mathrm{ff}$. 
lisionsentscheidung, den »Anknüpfungsbegriff « einer Vorbehaltsnorm (333). Er beschränkt sich auf den »Funktionalitätstest «, ob die nach den allgemein anerkannten Regeln der Rechtsmethodik vorzunehmende Anwendung (Konkretisierung) des einschlägigen Elements der vorbehaltenen Teilrechtsordnung (344) zur Verwirklichung der Policy dieser Teilrechtsordnung beiträgt (345). Voraussetzung eines solchen Funktionalitätstests ist die »Policy-Ermittlung «, für die es "kein allgemeingültiges Verfahren gibt« (34I). Gerade diese Offenheit soll aber den Anschluss an die Evolutionsfähigkeit von Teilrechtsordnungen gewährleisten: »Erst wenn sich eine Akzeptanz darüber, was ihre Policy ausmacht, herauszubilden vermag, kann davon die Rede sein, dass sie sich selbstorganisierend gestaltet und evolutionsfähig ist.«(342).

Die traditionelle juristische Methode soll also lediglich punktuell durch den Einbau der Erfahrung von Selbstorganisation und Evolutionsfähigkeit der Teilrechtsordnung, auf die eine Vorbehaltsnorm verweist, ergänzt werden. Methodisch ist eine Bezugnahme des Rechts auf soziale Standards, allgemein akzeptierte Werte und moralische Normen, die einem vom Recht nicht kontrollierten Wandel unterliegen, allerdings nichts Ungewöhnliches. Wo liegt - methodisch, nicht in der Sache - das gänzlich Neue der Bezugnahme auf die tragenden Elemente einer Teilrechtsordnung, wenn man etwa an die Formeln zur Konkretisierung von Treu und Glauben $\left(\mathbb{S} 242\right.$ BGB) oder guten Sitten ( $\int I_{3} 8$ BGB), an die in vielfältigen normativen Zusammenhängen geläufigen Verweisungen auf die Anschauungen aller billig und gerecht Denkenden, auf das ästhetische Empfinden eines Durchschnittsbetrachters in der Rechtsprechung zu den Verunstaltungsverboten des Bauordnungsrechts, auf die Anschauungen und Gebräuche der betroffe- nen Verkehrskreise und Ähnliches mehr denkt. Die punktuelle methodische Offenheit der Kollisionsentscheidung kann vielleicht mit der von Amstutz entfalteten Argumentation als Bedingung der Evolutionsfähigkeit des Rechts im Anschluss an die Evolution der Marktgesellschaft begründet werden. Aber damit entfällt nicht das Bedürfnis nach methodischen Vorkehrungen gegen beliebige und willkürliche Zugriffe des Richters auf das, was er für den in der Herausbildung einer Praxis akzeptierten Ordnungsanspruch einer Teilrechtsordnung halten mag.

Die Notwendigkeit des Einbaus von Erfahrung in die juristische Methode der Normauslegung und erst recht in den komplexen Vorgang richterlichen Entscheidens lässt sich meines Erachtens gerade nicht derartig punktuell begrenzen, wie dies Amstutz anzunehmen scheint. Er beschränkt sich auch nicht auf den Normenbestand des »kulturspezifischen Schutzmantels der Gesellschaft «. Im Gegenteil: Auch die traditionelle juristische Methodenlehre (und die universitäre Ausbildung von Juristen) übersieht immer noch allzu gerne, welche erkenntnistheoretische Problematik sich daraus ergibt, dass Normen in hohem Maße auf Tatsachenfeststellungen verweisen und dass die Auslegung von Normen bis zu dem Grad der Konkretisierung, der die Entscheidung des konkreten Falles erlaubt, auch von der Auswahl und Konkretisierung der Elemente des entscheidungserheblichen Sachverhalts abhängt. ${ }^{\text {I2 }}$ Bei erstinstanzlichen Zivilsachen sind zum Beispiel Auslegungsprobleme, die mehr als den schnellen Blick in den »Palandt « erfordern, die Ausnahme, Probleme bei der Bildung des entscheidungserheblichen Sachverhalts dagegen die Regel. So wird im Rahmen der Prüfung der Schlüssigkeit des Klägervorbringens ein Sachverhalt, im Rahmen der Prüfung der Erheblichkeit des Beklagtenvorbrin-

\footnotetext{
I 2 Friedrich Müller, Juristische Methodik, 7. Aufl. Berlin I997, I9of.
} 
gens ein anderer Sachverhalt und schließlich nach Beweiswürdigung ein dritter, nunmehr entscheidungstragender Sachverhalt gebildet. Alle drei Versionen müssen keineswegs mit der von einem Beobachter wahrgenommenen »Wirklichkeit « übereinstimmen. Gebildet werden diese Sachverhalte stets aus einer bestimmten normativen und prozessualen Perspektive. Gleichwohl gilt für die Parteien in den Grenzen der Dispositionsmaxime und für Zeugen und Sachverständige in den Grenzen der Aussageverweigerungsrechte die Wahrheitspflicht und für das Gericht im Rahmen der Prozessordnung die Maxime, auf die Feststellung des wahren Sachverhalts hinzuwirken. Auf die Probleme, die das in der Praxis bereitet, gehen Juristenausbildung und juristische Methodik kaum ein.

Der von der modernen Gehirnforschung und der Systemtheorie inspirierte Diskurs des radikalen Konstruktivismus ${ }^{\mathrm{I} 3}$ hat, auch wenn man die vorgeschlagenen Antworten nicht als überzeugend beurteilt, doch das Problembewusstsein dafür geschärft, dass die Bedeutung von Erfahrung für kognitive Konstruktionen mit einfachen Repräsentationsmustern nicht zureichend erfasst werden kann. Hier sei nur hingewiesen auf die zu wenig beachtete Erkenntnistheorie des späten Jean Piaget, die diese Zusammenhänge evolutionstheoretisch schlüssig modelliert. ${ }^{\mathrm{I} 4} \mathrm{Zu}$ untersuchen wäre, wie die Wahrnehmung von juristisch relevanten Sachverhalten gerade auch durch außerrechtliche soziale Kommunikation ermöglicht und zugleich begrenzt wird und wie auf diese Weise ein begrenztes, aber hinreichendes $\mathrm{Maß}$ an Übereinstimmung gestiftet wird. In der Argumentation der Studie von Amstutz zum operativen Modell des Rechts in der Marktgesellschaft finden sich dazu interessante Ansatzpunkte. Das Methodenkapitel schöpft dieses Potential aber nicht aus, weil auch das von Amstutz vorgeschlagene operative Modell letztlich keine Brücke zum Erkennen und Handeln sozialer Akteure findet.

Manfred Aschke

I3 Der Diskurs des Radikalen Konstruktivismus, hg. von SIEGFRIED J. SchMidT, Frankfurt am Main I987; Kognition und Gesellschaft. Der Diskurs des Radikalen Konstruktivismus 2, hg. von SIEGFRIED J. SCHMIDT, Frankfurt am Main I992.

I4 Jean PIaget, Die Äquilibration der kognitiven Strukturen, Stuttgart I976. 